\title{
Diabetes Mellitus at an Elderly Age
}

\author{
Authors \\ Andrej Zeyfang1 ${ }^{1}$, Jürgen Wernecke², Anke Bahrmann³
}

\author{
Affiliations \\ 1 Department of Internal Medicine, Geriatric Medicine, \\ Palliative Medicine and Diabetology, medius Klinik \\ Ostfildern-Ruit, Germany \\ 2 Department of Geriatric Medicine, Agaplesion \\ Diakonieklinikum Hamburg, Germany \\ 3 Department of Cardiology, Angiology and Pneumology, \\ University Hospital Heidelberg, Germany \\ published online $\quad 21.01 .2021$ \\ Bibliography \\ Exp Clin Endocrinol Diabetes 2021; 129: S20-S26 \\ DOI 10.1055/a-1284-6023 \\ ISSN 0947-7349 \\ (C) 2021. Thieme. All rights reserved. \\ Georg Thieme Verlag KG, Rüdigerstraße 14, \\ 70469 Stuttgart, Germany
}

German Diabetes Association: Clinical Practice Guidelines Diabetologie 2020; 15 (Suppl 1): S112-S119. DOI 10.1055/ a-1193-3879

\section{Correspondence}

PD Dr. med. Dr. Univ. Rom Andrej Zeyfang

Department of Internal Medicine

Geriatric Medicine

Palliative Medicine and Diabetology

medius Klinik Ostfildern-Ruit

Hedelfinger Straße 166

73760 Ostfildern

Germany

andrej.zeyfang@gmail.com

PD Dr. med. Anke Bahrmann

Department of Cardiology

Angiology and Pneumology

University Hospital Heidelberg

Im Neuenheimer Feld 410

69120 Heidelberg

Germany

anke.bahrmann@med.uni-heidelberg.de
About 3000000 people in Germany are over 65 years of age and have diabetes mellitus. In 2010, the prevalence of diabetes in the age group 80 years and older was $34 \%$ in women and $32 \%$ in men. The statements in the practical recommendations are mainly directed at the majority of elderly people with type 2 diabetes and can only reflect some of the special characteristics of elderly people with diabetes. Topics such as type 1 diabetes at an elderly age, end of life, interface management or ethics are covered in the complete $\mathrm{S} 2 \mathrm{k}$ guideline.

The geriatric patient is defined by multimorbidity and a higher age. Age-typical functional limitations and high vulnerability create a special need for action that goes beyond blood glucose control and the management of cardiovascular risk factors or diabetes-typical complications. For differentiated therapy planning, elderly patients should be divided into functional groups ( $\triangleright$ Table 1).

A geriatric assessment should be conducted to determine resources and deficits (division into functional groups) (Practice Tool 1, see Appendix).

\section{Treatment Objectives}

Maintaining quality of life and avoiding hypoglycemia are the primary therapeutic goals. Individual aspects of quality of life should therefore be actively investigated. The HbA1c value is less important in advanced age for therapy decisions and should also be evaluated from the point of view of presumed life expectancy.

General therapeutic goals regarding the improvement of quality of life are contained in Practice Tool 2 (see Appendix).

Individualized therapy goals should be agreed for the following parameters in elderly people with type 2 diabetes:

- Glucose metabolism

- Blood pressure

- Lipid profile

The focus is on preventing hypoglycemia ( Fig. 1). Lower HbA1c or pre- and postprandial values should only be targeted in therapies without a risk of hypoglycemia. The corresponding target corridors are shown in > Table 2.

\section{Hypoglycemia}

The risk of hypoglycemia increases with diabetes duration and is higher in elderly people with diabetes. The threshold for the perception of low blood glucose levels decreases with age; however, brain dysfunction already occurs at higher levels. In addition, the symptoms of hypoglycemia can take on a different form. 
- Table 1 Classification into functional groups.

\begin{tabular}{|l|l|}
\hline Patient group & Patient description \\
\hline Functionally independent & $\begin{array}{l}\text { Elderly people with diabetes mellitus and good functional status. Patients with low co-morbidity, possibly low } \\
\text { cognitive impairment and good compensation possibilities }\end{array}$ \\
\hline Functionally slightly dependent & $\begin{array}{l}\text { Elderly people with diabetes mellitus and limited functional status. Patients with multimorbidity, functional and } \\
\text { cognitive limitations and geriatric syndromes }\end{array}$ \\
\hline Functionally highly dependent & $\begin{array}{l}\text { Elderly people with diabetes mellitus and extremely limited functional status or terminally ill people. Patients with } \\
\text { multimorbidity, geriatric symptoms, pronounced functional and cognitive limitations and the presence of diseases } \\
\text { with limited life prognosis, e. g. terminal heart, kidney or malignant diseases }\end{array}$ \\
\hline End of Life & People who are on their deathbed \\
\hline
\end{tabular}

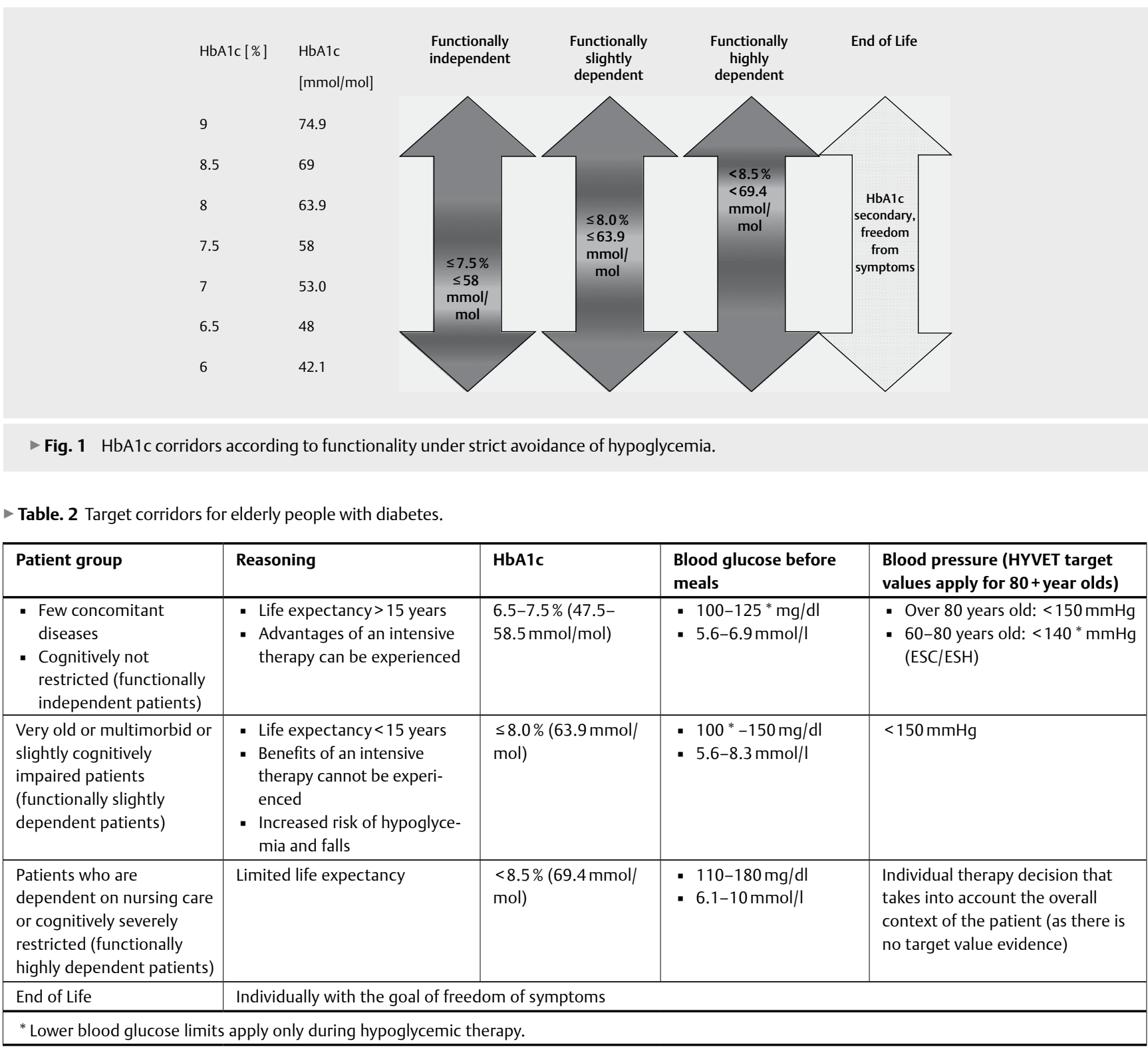

Hypoglycemia is the second most common cause of drug-related emergency admissions of elderly people. The incidence of severe hypoglycemia is very high, at $7.8 \%$ per patient per year in nursing homes.

Severe hypoglycemia is much more common in depression, kidney and heart failure and treatment with a beta-blocker. They are most common in treatments with prandial insulin, a basal insulin or insulin secretion; other antidiabetic treatments are associated with only a very low risk of hypoglycemia. Elderly people with concomitant diseases, multimorbidity or impaired cognitive performance are particularly at risk. 
Hypoglycemia is much more common in everyday life than previously thought, especially at night. Risk factors are long duration of illness, cognitive impairment and multimorbidity, especially renal failure. There is increasing evidence that hypoglycemia promotes the development of cardiovascular events and dementia in advanced age. The prevention of hypoglycemia is a primary therapeutic goal, especially for elderly people with diabetes. Therefore, the metabolic therapy goals and the implementation of therapy should be based on the functional resources and deficits of the patient.

Therapy forms with the lowest possible risk of hypoglycemia should be chosen.

\section{Hypertension}

- Elderly patients benefit from effective blood pressure reduction. This also applies to patients over 80 years of age in good physical and mental condition.

- There is only insufficient evidence for antihypertensive treatment in frail patients over 80 years of age, so that this therapy should be individualized (see $>$ Table $\mathbf{1}$ ).

- There is no evidence for the different efficacy of antihypertensive drugs in younger or elderly patients. All major antihypertensive classes can be recommended.

Due to frequent comorbidities and polypharmacy, special attention should be paid to known adverse drug reactions in elderly patients.

\section{Dyslipidemia}

- In elderly patients with diabetes as well as very high risk (e. g. CHD, severe kidney damage or one or more CV risk factors and/or organ damage) a target LDL cholesterol of $<1.8 \mathrm{mmol} / \mathrm{L}$ ( $<70 \mathrm{mg} / \mathrm{dL}$ ) or a LDL cholesterol reduction $\geq 50 \%$ should be aimed for.

- In elderly patients with diabetes without functional limitations (without other CV risk factors and without organ damage) a target LDL cholesterol of $<2.5 \mathrm{mmol} / \mathrm{L}(<100 \mathrm{mg} / \mathrm{dL}$ ) should be aimed for.

- For elderly patients with functional limitations, the use of statins should be evaluated on an individual basis.

- Statins should be used as first-line therapy to lower LDL cholesterol.

\section{Multi-medication}

- Prescription of more than five drugs increases the probability of occurrence of undesirable, clinically-relevant side effects/ interactions (e.g. severe hypoglycemia, comorbidity) and reduces patient adherence.

- The treatment regimen should be based on the patient's wishes, current quality of life and self-care abilities.

- The drug therapy should be reviewed critically on a regular basis.

\section{Assessment}

Geriatric assessment refers to functional tests that are available as a diagnostic tool for recording resources and deficits and quantify- ing them in geriatric patients. Implementation and evaluation, etc. see www.kcgeriatrie.de

\section{Secondary Diseases}

For diabetic secondary diseases, also in advanced age, the statements by the National Disease Management Guidelines (NVL) on diabetes and retinopathy, nephropathy, diabetic foot syndrome and neuropathy apply in principle. Some particularities in elderly people are highlighted below as examples:

\section{Diabetic foot}

- Diagnostics: in contrast to monofilament examination, the examination of vibration sensation with the 128-Hertz tuning fork can be omitted in patients of advanced age.

- Therapy: the multi-professional geriatric team is an indispensable addition in the treatment of elderly and geriatric patients with diabetic foot syndrome.

- Prophylaxis: when selecting footwear, the risk of falling should be taken into account, e. $\mathrm{g}$. in patients at risk of falling, metatarsal bars should not be used.

\section{Coronary heart disease (CHD)}

- Revascularization of coronary vessels in elderly diabetic patients remains a challenge due to the lack of randomized studies comparing percutaneous coronary intervention ( $\mathrm{PCl})$ with bypass surgery, especially in elderly people with diabetes.

- Meta-analyses suggest that PCI is beneficial in elderly people with diabetes with single-vessel disease, while bypass surgery is the better choice in triple-vessel disease.

\section{Acute coronary syndrome}

- Diabetes with acute coronary syndrome is associated with a lower rate of successful coronary revascularization and the risk of periinterventional complications in elderly people compared to younger people.

- Insulin therapy should be started in patients with hyperglycemia $>10 \mathrm{mmol} / \mathrm{l}$ in the acute phase of acute coronary syndrome, taking into account the comorbidities.

\section{Stroke}

- In the context of an acute stroke, older diabetes patients with blood glucose levels $>198 \mathrm{mg} / \mathrm{dl}$ (>11 mmol/l) should be consistently treated with insulin, as there is evidence that hyperglycemia increases stroke volume and is associated with a poorer functional outcome.

- In a large randomized study, intensified insulin therapy with only slightly to moderately-elevated serum glucose levels (median $137 \mathrm{mg} / \mathrm{dl}(7.6 \mathrm{mmol} / \mathrm{l})$ ) had no effect on mortality and functional outcome in patients and cannot be recommended in general, especially due to the risk of hypoglycemic episodes. 


\section{Therapy}

Non-drug treatments are also the basis of treatment for elderly people with diabetes. In addition to the recommendations of the NVL, there are particularities:

\section{Training}

Elderly people with diabetes should also participate in structured diabetes training. A special evaluated training program (SGS) is currently available for the group of functionally-impaired patients and is also available in many foreign languages. If possible, relatives and friends should also participate in such a program.

\section{Movement and tendency to fall}

- Elderly people with diabetes mellitus should be checked for a possible risk of falling.

- In elderly people with diabetes and an increased risk of falling, medication should be reviewed with regard to drugs that increase the tendency to fall (e.g. neuroleptics, hypnotics, sedatives).

- Elderly people with diabetes should have their visual acuity regularly checked and, if necessary and possible, an offer for correction should be made.

- Functionally mildly or severely-impaired elderly people with diabetes and their relatives should be made aware of possibilities for fall prevention and of fall hazards at home (lack of lighting, missing handles in the bathroom and near the toilet, etc.)

- Functionally mildly or severely-impaired elderly people, especially those with frailty and diabetes, should be offered opportunities for strength and endurance training.

- Exercise is considered the most important lifestyle measure for the prevention of dementia in advanced age.

\section{Nutrition}

- The consequences of malnutrition are serious in advanced age. Elderly people who are overweight or obese should avoid strict dietary restrictions due to the risk of malnutrition.

- The risk of malnutrition can be assessed using a Mini Nutritional Assessment (MNA).

- In underweight patients, the causes should be clarified by a multi-professional team.

\section{Oral antidiabetics and GLP-1 agonists}

Advantages mentioned for the individual substances apply to monotherapy. In combination with insulin or insulinotropic substances (e. g. sulfonylureas, glinides), the increased risk of side effects (e. g. risk of hypoglycemia) should be taken into account. Combinations of $>2$ OADs should be avoided in view of the risks of polypharmacotherapy in advanced age.

Acarbose, glitazones, sulfonylureas and glinides are considered by the majority to be less suitable. For use in advanced age, the FORTA recommendations (Fit for The Aged) can also be consulted.

\section{Metformin}

Metformin is also to be used as a drug therapy of first choice for elderly patients, although clinical studies on the administration of metformin in advanced age are not available (FORTA Recommen- dation B). If metformin is used, patients should be carefully advised to discontinue/pause metformin in situations where there is a risk of acute deterioration in kidney function (examination with X-ray contrast agents, surgery under general anesthesia, febrile diseases, exsiccosis or gastrointestinal infections). Metformin can be prescribed up to a glomerular filtration rate of $>30 \mathrm{ml} / \mathrm{min}$ in a maximum dose of $1000 \mathrm{mg}$, divided into two single doses. Vitamin B 12 deficiency may occur after prolonged metformin intake.

\section{DPP-4 inhibitors}

DPP-4 inhibitors can be used in elderly patients, according to FORTA first choice (Recommendation A) for elderly patients. Advantages can be seen in particular with regard to therapy adherence (low frequency of administration required), risk of hypoglycemia, weight neutrality and higher degree of renal failure (dose reduction).

\section{GLP-1 agonists}

GLP1 agonists can be used in elderly patients in individual cases (FORTA Recommendation B). Advantages can include: low risk of hypoglycemia, weight loss - if this is also the aim of therapy; for liraglutide and dulaglutide: reduced cardiovascular morbidity and mortality; for dulaglutide and long-acting exenatide: administration once a week.

\section{SGLT-2 inhibitors (gliflozin)}

SGLT-2 inhibitors can be used in elderly patients. The start of therapy with dapagliflozin is not recommended after the age of 75 years; for empagliflozin a limit of 85 years applies. Advantages can include: lowering of blood glucose levels without personal risk of hypoglycemia and reduced cardiovascular morbidity and mortality, avoidance of hospitalization due to heart failure as well as a nephroprotective effect in patients with type 2 diabetes. During use, increased risks, e.g. for urogenital infections, polyuria, exsiccosis and deterioration of renal function as well as the (rare) occurrence of (normoglycemic) ketoacidosis should be noted.

For metformin and SGLT-2 inhibitors, the recommendation to pause the medication in times of increased stress (as with taking "sick days") has proven to be effective.

\section{Insulin}

- Insulin therapy should be started if lifestyle changes and/or oral antidiabetics do not achieve the individualized therapy goal or oral antidiabetics are no longer effective due to contraindications or if polypharmacy can be reduced.

- Before insulin therapy, it should be decided whether insulin should be given as monotherapy or in combination with oral antidiabetics. The choice of insulin therapy depends primarily on the patient's wishes, cognitive and fine motor skills as well as the social environment and the therapeutic goal.

The following particularities apply especially to geriatric patients: Short-acting insulins (normal and analogue insulins):

- For safety reasons, all short-acting insulins can be given after the start of a meal without an injection-to-meal interval or in the case of loss of appetite or dementia. Long-acting insulins (NPH and analogue insulins): 
- The rate of nocturnal hypoglycemia is lower among analogue basal insulins with a normoglycemic therapy target than among NPH insulins. Cloudy NPH insulins have the disadvantage that they must be mixed well before injection.

- Provided that the patient consumes regular meals and exercises, mixed insulins can be used if fewer injections and blood glucose tests are desired.

- Insulin degludec can be injected at variable injection times, which makes it easier to administer e. g. by nursing services.

The care of patients in domestic and long-term inpatient care

Complex insulin therapy regimens are often used at an advanced age with a too strict setting. At the same time, there is insufficient communication between general practitioners and nurses regarding insulin therapy, and emergency instructions are rarely available.

\section{Technology}

Technical aids for diabetes management for the elderly have been on the market for many years. While mechanical aids such as ageappropriate blood glucose meters or age-appropriate insulin pens are already well-established and well-accepted, electronic aids (e.g. pillboxes with reminder function) or digital aids (PC programs or apps) have not yet found widespread use.

The evaluation of individual aids can be found in Practice Tool 3 (see Appendix).

\section{BG self-monitoring}

Blood glucose self-monitoring should be carried out individually and within the scope of the possibilities (self-care competence) of the patient and the relatives caring for him/her.

\section{Geriatric Syndromes and Special Situations in Advanced Age}

\section{Frailty and sarcopenia}

Frailty and sarcopenia are two geriatric syndromes which are of great importance for the preservation of functionality, independence and quality of life of the elderly.

Frailty is a multidimensional syndrome characterized by decreased functional reserves, reduced resistance to stresses and increased vulnerability to negative health events such as falls, institutionalization and mortality. Frailty is diagnosed on the basis of five criteria (Practice Tool) that describe a physical phenotype.

Sarcopenia is the age-associated loss of muscle mass and muscle function, often associated with weakness and the risk of falling. Both occur more frequently in elderly people with diabetes than in the population without diabetes.

- Sarcopenia/frailty screening should be performed in elderly patients with diabetes.

- An optimized diet and adapted training programs should be the basis of therapy for sarcopenia and frailty.

- In patients with an increased rate of falls and fractures, measures should be taken to prevent falls.

\section{Depression}

Depression is a common mental disorder among elderly people with diabetes. A bidirectional relationship is known and also a mutual negative influence on physical activity, compliance/adherence or dementia. With some limitations, elderly patients with diabetes can be treated in the same way as younger patients.

- Elderly people with diabetes should be screened for depression once a year. If the result is positive, a validated, age-specific procedure should be performed.

- Elderly people with diabetes and depression should pay particular attention to signs of possible suicidal tendencies due to the increased risk of suicide.

- Interventions that promote physical activity and social contacts should be used in treating depression.

\section{Dementia}

In Germany there are currently about 1700000 people with dementia. People with type 2 diabetes have a higher risk of dementia in advanced age compared to people without diabetes. Dementia has a negative impact on other geriatric syndromes, especially frailty, and is associated with poorer metabolic control and an increased risk of hypoglycemia.

- In elderly people with diabetes, a screening of cognitive performance should be performed once a year using a validated method.

- The cognitive requirements of diabetes treatment should be adapted to the cognitive performance of the person with diabetes.

- Training measures should be adapted to the cognitive abilities of people with diabetes and the complexity of the content and level of language taught should be adapted accordingly.

- Activating the body and encouraging physical activity should be encouraged within the existing physical abilities of an elderly person with diabetes and dementia.

\section{Urinary incontinence}

- Diabetic cystopathy is often associated with diabetic neuropathy. Polyneuropathy should therefore always be a reason for the examination of bladder function.

- Elderly people with diabetes should have an incontinence assessment at least once a year.

- Indwelling catheters should be avoided if possible; an indication for an indwelling catheter for urinary incontinence is only given if all other options fail, are rejected or cannot be applied.

\section{Special Features in Care}

- The planning and evaluation of care should always be evidence-based and should incorporate current expert standards German Network for Quality Development in Nursing [DNQP] and interdisciplinary care guidelines Association of the Scientific Medical Societies in Germany [AWMF].

- The situation and risk assessment should always be carried out together with the family doctor and all professional groups involved in the care. 
- Screening/assessment tools should be shared in the exchange of information across the different areas of care (primary care, hospital, outpatient and long-term inpatient care, etc.).

- The care focus of geriatric patients with diabetes should include the following: avoidance of hypoglycemia, prevention of diabetic foot syndrome, recording of pain (especially with regard to polyneuropathies), monitoring of the skin, wound assessment, wound management and promotion of oral health, recording of the nutritional situation, preservation and promotion of continence and recording of mobility restrictions/danger of falling.

- Changes in the care needs of vulnerable geriatric patients with diabetes should be communicated immediately and in a standardized way to the subsequent care levels/providers (managing the transition of care) in order to seamlessly integrate the adaptation of medical, nursing and therapeutic services.

- Nursing documentation should always be used in joint case planning across occupational groups.

- When taking oral antidiabetics, the timing should be chosen to match the prescribed drug/group of drugs.

- When insulin or insulinotropic drugs (especially sulfonylureas) are administered, sufficient food intake should be ensured to avoid hypoglycemia.

- In order to ensure subcutaneous insulin injection, the cannula length should be selected to suit the patient's constitution.

- Pen needles should not be used more than once.

- To avoid lipohypertrophy, the (varying) injection site should be determined in a rotation plan.

- The injection site should be chosen with regard to the speed of insulin absorption, any skin lesions or lipohypertrophies and recorded in a rotation plan.

- Elderly people with diabetes who carry out their insulin therapy and metabolic control themselves should be regularly assessed for their cognitive and sensorimotor abilities, especially when they are adjusting or changing their therapy. Certain tests from geriatric assessment are suitable for this purpose.

- Training content should be coordinated across the different levels of care, but above all between the hospital, family doctor and home care, and thus jointly safeguarded.

The proportion of diabetes patients among the elderly of at least $25-30 \%$ is very high across all care facilities, outpatient services, homes and clinics. At the same time, access to diabetological expertise for this patient group is low and not guaranteed for all regions. In order to ensure that diabetes patients in need of care are provided with guideline-based care no matter their location, a separate training pillar "Diabetes Care" has been added to the training program of the German Diabetes Society (DDG). The continuing education pillar consists of a basic qualification as well as continuing education to become a DDG diabetes care specialist with additional designation of the field of activity "long-term" or "clinical" (see www.deutsche-diabetes-gesellschaft.de).

\section{Appendix: Practice Tools}

\section{Practice Tool 1 Test procedure of geriatric assessment and its importance for the domains of diabetes treatment}

\begin{tabular}{|c|c|c|c|}
\hline Area & Particularities & Test procedure & $\begin{array}{l}\text { Short and } \\
\text { practical }\end{array}$ \\
\hline Training & $\begin{array}{l}\text { Cognition testing, } \\
\text { special curriculum/ } \\
\text { media required, } \\
\text { training of relatives } \\
\text { and caregivers/ } \\
\text { nursing care staff }\end{array}$ & $\begin{array}{l}\text { Visual acuity, } \\
\text { hearing, clock- } \\
\text { completion test, } \\
\text { Mini Mental State } \\
\text { Examination (MMSE) } \\
\text { (acc. to Folstein), } \\
\text { timed test of money } \\
\text { counting, DemTect, } \\
\text { Geriatric Depression } \\
\text { Scale (GDS) }\end{array}$ & $\begin{array}{l}\text { Clock-com- } \\
\text { pletion test, } \\
\text { timed test of } \\
\text { money } \\
\text { counting }\end{array}$ \\
\hline Movement & $\begin{array}{l}\text { Exercise therapy } \\
\text { made difficult, } \\
\text { realistic recom- } \\
\text { mendations } \\
\text { (walking) useful, } \\
\text { effects on bones, } \\
\text { fall/fractures, } \\
\text { cognition }\end{array}$ & $\begin{array}{l}\text { Timed Up \& Go, } \\
\text { five-chair-rise, SPPB, } \\
\text { Tinetti Test }\end{array}$ & $\begin{array}{l}\text { Timed Up \& } \\
\text { Go }\end{array}$ \\
\hline Nutrition & $\begin{array}{l}\text { Frequent } \\
\text { malnutrition in } \\
\text { advanced age, } \\
\text { usual nutritional } \\
\text { recommendations } \\
\text { (whole grain, } \\
\text { losing weight) not } \\
\text { reasonable, } \\
\text { realistic recom- } \\
\text { mendations } \\
\text { necessary for } \\
\text { everyday life }\end{array}$ & $\begin{array}{l}\text { Condition of } \\
\text { dentures, } \\
\text { endoscopic } \\
\text { evaluation of } \\
\text { swallowing, } \\
\text { Mini-Nutritional } \\
\text { Assessment (MNA), } \\
\text { NRS } 2002\end{array}$ & $\begin{array}{l}\text { MNA } \\
\text { Screening }\end{array}$ \\
\hline $\begin{array}{l}\text { Pharmaco- } \\
\text { therapy }\end{array}$ & $\begin{array}{l}\text { Consider } \\
\text { polypharmacy and } \\
\text { susceptibility to } \\
\text { iatrogenic damage, } \\
\text { compliance is } \\
\text { determined by } \\
\text { cognition and } \\
\text { depression, use } \\
\text { insulin when } \\
\text { necessary }\end{array}$ & $\begin{array}{l}\text { Check of the ability } \\
\text { to take medication, } \\
\text { timed test of money } \\
\text { counting }\end{array}$ & \\
\hline
\end{tabular}

\section{Practice Tool 2 General therapy goals in terms of improving the quality of life}

- Avoidance of acute complications (especially hypoglycemia)

- Minimization of the side effects of the therapy (e.g. severe hypoglycemia) and of stress on the patient caused by the therapy (avoidance of polypharmacy)

- Reduction of geriatric syndromes

- Increasing the competence (empowerment) of those affected in dealing with the disease

- Reduction of a disease stigma

- Treatment satisfaction

- Promotion of therapeutic adherence through individuallyadapted therapy 
- Regular monitoring of the understanding of therapy (cognitive, sensorimotor, psychological) and the individual options

- Prevention and treatment of symptoms by improving the metabolic control

- Treatment and improvement of comorbidities

- Avoidance of overtreatment by reducing polypharmacy where possible

- Avoidance of overdiagnosis, where possible

\section{Practice Tool 3 Technology for elderly people}

Mechanical aids:

- Magnifying glasses and other visual aids, speaking BG measuring device (very suitable)

- BG meters which do not require calibration, with a large display and easy operation (very suitable)

- Insulin pens with easy release and low thumb pressure (very suitable)

- Step-by-step instructions integrated into everyday objects (very suitable)

- Pillboxes with weekly supply (very suitable)

Technical aids for elderly people with diabetes in a broader sense:

- Automatic blood pressure monitors, preferably with upper arm cuff and electric pump

- Walking aids for polyneuropathy and/or frailty (e. g. walking frame, walker)

- Fracture prevention (e.g. safe hip protection pants, anti-slip socks)
Electronic aids:

- PC programs for analyzing collected measured values and data (partly suitable)

- Apps to improve therapy compliance (partially suitable)

- Apps for data management and BG control (partly suitable)

- Technical aids to remind patients to take medication or inject insulin (partially suitable)

- Automatic lighting with motion sensors to prevent falls (very suitable)

- Sensor mats or radio frequency identification (RFID)/Global Positioning System (GPS) systems to improve safety, for example in the case of dementia (limited suitability)

- Flash glucose monitoring (FGM) sensors are easy to use even for elderly people with diabetes and can lead to improved blood glucose control and better quality of life.

- Continuous glucose monitoring (CGM) systems are only suitable for some elderly people due to more complex operation

\section{Conflict of interest}

$A B$ received honoraria for presentations from Bayer, Böhringer Ingelheim, Pfizer, Daiichi Sankyo, Lilly, NovoNordisk and Novartis. AZ received fees for lectures from Berlin-Chemie, Boehringer-Ingelheim, Lilly, Novo-Nordisk and Sanofi-Aventis. JW has received honoraria for lectures from Boehringer Ingelheim, Chiesi, Lilly, MSD, Novartis, Novo-Nordisk and Sanofi-Aventis. 